Gynäk. Rdsch. 1969;7:I-VII

\title{
Contents, Vol. 7, 1969
}

\section{Übersichtsaufsätze}

Nogales, F.; Parache, J. und Martinez, H.: Pathologische Anatomie der Genitaltuberkulose. Bericht über 1205 Fälle 81

Wright, S. W. und Sparkes, R. S.: Genetische Beratung bei Schwachsinn .... 241

Kurzfassungen von Zeitschriftenartikeln

Agfalvi, Rosza: vide Rosta, J.

Albright, N.: vide Altchek, A.

Altchek, A.; Albright, N. und Sommers, S.: Die Pathologie der Niere bei Schwangerschaftstoxikosen 228

Amsterdam, D.: vide Horowitz, M. I.

Assali, N. S. und Morris, J. A.: Kreislauf- und metaboiische Anpassung des Fetus bei der Geburt 23

Baric, L.: vide Wakefield, J.

Beneke, G.: Mammatumoren 49

Berkovich, S. und Pangan, J.: Virusuntersuchungen an frühgeborenen Kindern während des gehäuften Auftretens von Erkrankungen der Luftwege, unter besonderer Berücksichtigung des ECHO-Virus Typus 22276

Bettleheim, K. A.: vide Spencer, A. G.

Brancato, F.: vide Freeman, R. B.

Bromer, L.: vide Freeman, R. B.

Brown, W. E.: vide Clark, R. B.

Buckels, L. J. und Usher, R.: Cardiopulmonaler Effekt der plazentaren Trans fusion 220

Buckle, A. E. R. und Hills, A. M. E.: Erfahrungen mit dem Intrauterinpessar . . 41

Cahen, R. L.: Bestimmung der teratogenen Wirkung von Pharmaka 19

Clark, R. B.; Cooper, J. O.; Stephens, S. R. und Brown, W. E.: Untersuchungen

zum Säure-Basenhaushalt Neugeborener 278

Cohen, S. I.: vide Freeman, R. B

Cooper, J. O.: vide Clark, R. B.

Dale Poole, B.: Geistige Retardierung und Hilfsprogramm für Mutter und Kind . 144

Donaldson, LA.: Abnorme Positionen derNabelschnur. Eine Diskussion überpe-

rinatale Gefahren 218

Duncan, B. R.: vide Frankenburg, W. K.

Eden, A. N.: Perinatale Mortalität infolge einer Jnfektion mit Vibrio fetus .... 133

Egger, I.: vide Werner, $\mathrm{H}$.

El-Mallahawi, M. N.; Seif El-Din, D.; Mahran, M.; Sabour, M. S. und Fadel, H. E.: Die glomeruläre Filtrationsrate in der normalen Schwangerschaft und der frühen postpartalen Periode 119 d'esopo, D. A.: vide King, T. M. 
Esquivel, A. und Laufe, L. E.: Antikonzeption mittels einer monatlichen Einzelinjektion 36

Evans, A. T.: vide Hoodin, A. O.

Eykyn, S. J.: vide McFadyen, I. R.

Fadel, H. E.: vide El-Mahallawi, M. N.

Fine, R. N.: vide Fosson, A. R.

IV

Inhaltsverzeichnis

Fosson, A. R. und Fine, R. N.: Neugeborenen-Meningitis. Darstellung und Diskussion von 21 Fallen 222

Frankenburg, W. K.; Duncan, B. R.; Wendell Coffelt, R.; Koch, R.; Gold-

well, J. G. und Son, C. D.: Mütterliche Phenylketonurie: Folgen für Wachstum und Entwicklung 282

Freeman, R. B.; Bromer, L.; Brancato, F.; Cohen, S. I.; Garfield, C. F.; Griep, R. J.; Hirnman, E. J.; Richardson, J. A.; Thurm, R. H. und Urner, C.:

Verhütung einer rekurrierenden Bakteriurie durch kontinuierliche Chemotherapie $\quad 113$

Futrakul, P.: vide Miller, H. C.

Garfield, C. F.: vide Freeman, R. B.

Goldwell, J. G.: vide Frankenburg, W. K.

Griep, R. J.: vide Freeman, R. B.

Gruenwald, P.: Fetales Wachstum als Massstab für die Änderung im sozio-

ökonomischen Status 214

Hammond, J.: vide Wood, C.

Hellmich, E.: Der Einfluss von Frauenmilch- und Kuhmilchernährung auf den

Icterus neonatorum Frühgeborener 268

Hills, A. M. E.: vide Buckle, A. E. R.

Hirman, E. J.: vide Freeman, R. B.

Hirshleifer, i.: vide Horowitz, M. I.

Hoodin, A. O.; Malin, J. M. und Evans, A. T.: Ungewöhnliche Komplikationen

nach urethro-vesicaler Suspension wegen Urin-Inkontinenz 121

Horowitz, M. I.; Amsterdam, D. und Hirshleifer, 1.: Zur Methodik der Beurteilung von antimikrobiellen Harndesinfizientien 288

John, A. H.: Der Effekt der mütterlichen Hypoxie auf die Herzfrequenz des Fetus in utero 132

Kappas, A.: Biologische Wirkungen einiger natürlicher Steroide auf die Leber . . . 43

Katz, R. G.; White, L. R. und Sever, J. L.: Röteln in der Schwangerschaft . . 258

Kelly, S. und Rose, F.: Der Nachweis von Phenylketonurieüberträgern 283

Keuth, U. und Moeller, B.: Zur unzulänglichen Erfassung des Morbus haemo-

lyticus neonatorum 263

King, T. M. und d'Esopo, D. A.: Gehirntumoren in der Schwangerschaft .... 213

Kloos, K. und Vogel, M.: Plazentationsstörungen 197

Koch, R.: vide Frankenburg, W. K.

Koya, Y.: Bleibt der Effekt eines Programme für Familienleben bestehen? .... 150

Kunin, C. M. und Paquin, A. J.: Harnwegserkrankungen bei Schulmädchen mit

Bakteriurie 110 
Kuusisto, P.: vide Timonen, S.

Laufe, L. E.: vide Esquivel, A.

Leck, J. und Rogers, S. C.: Häufigkeitsschwankungen beim Auftreten des Anenzephalus $\quad 16$

Lemieux, G. und Martin, M. St. : Die Zuverlässigkeit des Mittelstrahlurins zur

Diagnose der Bakteriurie der Frau 103

Lemtis, H.: Die Plazentation des Menschen unter besonderer Berücksichtigung der materno-fetalen Kreislaufbeziehungen (Schematische Skizzen) 179

Levison, H. und Swyer, P. R.: Sauerstoffaufnahme und die thermische Umgebung des neugeborenen Kindes 280

Liggins, G. C. und Whittington, E.: Eine Routinemethode zur Feststellung einer

Schwangerschaftsbakteriurie 102

Lokki, O.: vide Timonen, S.

Lumley, J.: vide Wood, C.

Inhaltsverzeichnis $\mathrm{V}$

Mahran, M.: vide El-Mahallawi, M. N.

Malin, J. M.: vide Hoodin, A. O.

Manabe, Y.: vide Mastroianni, L., Jr.

Martin, M. St.: vide Lemieux, G.

Martinez, H.: vide Nogales, F.

Mastroianni, L., Jr.; Suzuki, S.; Manabe, Y. und Watson, F.: Weitere Beobachtungen über den Einfluss des Intrauterinpessars auf die Eizelle und die Spermienverteilung beim Affen 39

McFadyen, 1. R. und Eykyn, S. J.: Suprapubische Aspiration von Urin während der Schwangerschaft 109

Meisels, A.: Die Menopause: Eine zytohormonale Untersuchung 31

Miller, H. C. und Futrakul, P.: Geburtsgewicht, Tragzeit und Geschlecht Frühgeborener als entscheidende Faktoren beim Zustandekommen des 'Respiratory Distress Syndrome’ 274

Moeller, B.: vide Keuth, U.

Morris, J. A.: vide Assali, N. S.

Mulcahy, D.: vide Spencer, A. G.

Newman, W.: vide Wood, C.

Nogales, F.; Martinez, H. und Parache, J.: Abstossung und Wiederaufbau des

menschlichen Endometriums 292

Notter, A.: Schmerzlose Geburt durch Psycho-Physioprophylaxe 161

O’Grady, F. W.: vide Spencer, A.G.

Pangan, J.: vide Berkovich, S.

Paquin, A. J.: vide Kunin, C. M.

Parache, J.: vide Nogales, F.

Prechtl, H. F. R.: Neurologische Folgen prä- und perinataler Komplikationen . 8

Reyn, A.: Die Laboratoriumsdiagnostik der Gonorrhoe 208

Richardson, J. A.: vide Freeman, R. B.

Rogers, S. C.: vide Leck, J.

Rose, F.: vide Kelly, S.

Rosta, J.; Szöke, L. und Agfalvi, Rosza: Der Bilirubinspiegel im Nabelschnurblut 
und der Blutaustausch im Neugeborenenalter

Sabour, M. S.: vide El-Mallahawi, M. N.

Salzman, E. W.: Kommt es beim hämorrhagischen Schock zur intravaskulären Blutgerinnung? 285

Samples, D. M. und Weed, J. C.: Gynäkologische Aspekte der idiopathischen

thrombopenischen Purpura. Erfahrungen aus 29 Fallen 212

Seale, J. R.: Geschlechtskrankheiten in der Ehe 210

Sealy, W. C.: Die Lunge im hämorrhagischen Schock 286

Self El-Din, D.: vide El-Mallahawi, M. N.

Sever, J. L.: vide Katz, R. G.

Shooter, R. A.: vide Spencer, A. G.

Silverman, W. A.: vide Sinclair, J. C.

Sinclair, J. C. und Silverman, W. A.: Intrauterines Wachstum der aktiven Zell-

masse des menschlichen Fetus mit besonderer Berücksichtigung des untergewich-

tigen Neugeborenen 270

Sinnathuray, T. A.: Vorkommen und Signifikanz der Nabelschnurumschlingung . 29

Son, C. D.: vide Frankenburg, W. K.

Spencer, A. G.; Shooter, R. A.; Bettleheim, K. A.; Mulcahy, D.; O’Grady,

F. W. und Taylor, J.: Escherichia-coli-Serotypen bei Harntraktinfektionen . . 106

Schäfer, V.: Der Infektabwehrmechanismus der Harnblase 108

Stephens, S. R.: vide Clark, R. B.

VI

Inhaltsverzeichnis

Stern, C.: Einige allgemeine Aspekte der Humangenetik 1

Suzuki, S.: vide Mastroianni, L.

Swyer, P. R.: vide Levison, H.

Szöke, L.: vide Rosta, J.

Taylor, J.: vide Spencer, A. G.

Thurm, R. H.: vide Freeman, R. B.

Timonen, S.; Uotila, U.; Lokki, O.; Kuusisto, P. und Vara, P.: Abschätzung der

wahrscheinlichen Schwangerschaftsdauer aufgrund der Reifezeichen des Kindes 147 Tomlin, E.

M.: vide Youngblood, V. H. Tovell, H. M. M.: vide Van Praagh, 1. G. L. Towbin, A.:

Ventrikelblutungen und Infarkte der subependymalen Matrix bei Feten

und unreifen Neugeborenen 134

Tünte, W.: Zur Frage der jahreszeitlichen Häufigkeit der Anencephalie

Urner, C.: vide Freeman, R. B.

Uotila, U.: vide Timonen, $\mathrm{S}$.

Usher, R.: vide Buckels, L. J.

Van Praagh, I. G. L. und Tovell, H. M. M.: Kaiserschnitt aus kindlicher Indi-

kation 27

Vara, P.: vide Timonen, S. Vogel, M.: vide Kloos, K.

Wachtel, E.: Die Zytologie der Amenorrhoe 33

Wakefield, J. und Baric, L.: Die Meinung von Fachleuten und Öffentlichkeit über

ein «screening »-Programm zur Früherfassung des Cervix-Carcinoms

Watson, F.: vide Mastroianni, L., Jr.

Weed, J. C.: vide Samples, D. M. 
Weghaupt, K.: Das Vulvakarzinom und seine Behandlung. Ein Bericht aus den Jahren 1952 bis 1962204

Wendell Coffelt, R.: vide Frankenburg, W. K.

Werner, H. und Egger, I.: Die latente Toxoplasma-lnfektion des Uterus und ihre Bedeutung für die Schwangerschaft 259

White, L. R.: vide Katz, R. G.

Whittington, E.: vide Liggins, G. C.

Wolfe, J. N.: Untersuchungen des Brustgewebes mit Hilfe der Mammographie bei gesunden Frauen mit gutartigen oder bösartigen Brusterkrankungen 151

Wood, C.; Lumley, J.; Hammond, J. und Newman, W.: Die Erkennung fetaler Gefahrenzustände unter der Geburt bei Patientinnen mit Hypertonie 261

Youngblood, V. H. und Tomlin, E. M.: latrogene Blasendysfunktion bei der Frau 122 Kasuistik

Babenerd, J.: Die hyperbare Sauerstoff-Therapie bei Gasbrandinfektionen in der Frauenheilkunde 235

Aus der Geschichte der Geburtshilfe

Müller, C. und Portmann, M. L.: William Smellie (1697-1763) und sein Beitrag zur modernen Geburtshilfe 154

Varia Ostrogene

Referate, gehalten am 15. Symposium der Deutschen Gesellschaft für Endokrinologie, Köln, 6.-8. März 1968123

Inhaltsverzeichnis VII

Gesundheit von Mutter und Kind

Maternal and Child Health, Apha Conference Reports 1965: Ärzte müssen Nutzen und Gefahren von Medikamenten kennen 256

Bericht der Konsiliarpädiater und der Medical Officers of Health der südostschottíschen Krankenhausregion: Kontrolle der Bevölkerung durch den Guthrie-Test auf Phenylketonurie in Südostschottland 284 